\title{
A STABILITY CONDITION FOR $n$th ORDER DIFFERENCE EQUATIONS
}

\author{
by RUSSELL A. SMITH
}

(Received 17 September, 1969)

1. Introduction. Consider the system of difference equations

$$
x(t+n)+a_{1} x(t+n-1)+a_{2} x(t+n-2)+\ldots+a_{n} x(t)=0,
$$

in which the unknown $x(t)$ is a complex $m$-vector, $t$ is a real variable and $a_{1}, \ldots, a_{n}$ are complex $m \times m$ matrices whose elements are functions of $t, x(t), x(t+1), \ldots, x(t+n-1)$. A positive definite hermitian form $V\left(x_{1}, x_{2}, \ldots, x_{n}\right)$, with constant coefficients, is called a strong autonomous quadratic Lyapunov function (written strong $A Q L F$ ) of (1) if there exists a constant $\kappa>1$ such that $\kappa^{2} v(t+1)<v(t)$ for all non-zero solutions $x(t)$ of $(1)$, where $v(t)=V(x(t)$, $x(t+1), \ldots, x(t+n-1))$. The existence of a strong AQLF is a sufficient condition for the trivial solution $x=0$ of (1) to be globally asymptotically stable. It is a necessary condition only in the special case of an equation

$$
x(t+n)+c_{1} x(t+n-1)+c_{2} x(t+n-2)+\ldots+c_{n} x(t)=0,
$$

in which the matrices $c_{1}, \ldots, c_{n}$ are constant. The variable matrices $a_{1}, \ldots, a_{n}$ in (1) will be assumed to satisfy

$$
\left\|a_{v}-c_{v}\right\| \leqq \delta_{v} \quad(v=1, \ldots, n),
$$

for all $t, x(t), \ldots, x(t+n-1)$, where $\delta_{1}, \ldots, \delta_{n}$ are constants. Here $\|M\|$ denotes the spectral norm of the matrix $M$, which is defined to be the square root of the largest eigenvalue of $M^{*} M$, where $M^{*}$ is the conjugate transpose of $M$. When $\delta_{1}, \ldots, \delta_{n}$ are small, (1) can be regarded as a perturbation of (2). It is not difficult to show that, if (2) has a strong AQLF, then (1) also has a strong AQLF, provided that $\delta_{1}, \ldots, \delta_{n}$ are sufficiently small. The main object of this paper is to replace these words " sufficiently small " by an explicit estimate which is simple and in some cases best possible. To ensure that (2) has a strong AQLF, it will be assumed that its characteristic polynomial $p(\zeta)$ can be factorised in the form

$$
p(\zeta)=\zeta^{n}+\sum_{v=1}^{n} c_{v} \zeta^{n-v}=\left(\zeta e-k_{n}\right)\left(\zeta e-k_{n-1}\right) \ldots\left(\zeta e-k_{1}\right)
$$

where $e$ is the unit $m \times m$ matrix and $k_{1}, \ldots, k_{n}$ are matrices having

$$
\left\|k_{v}\right\|<1 \quad(v=1, \ldots, n) .
$$

This assumption reduces the generality of (2) when $m>1$, because some matrix polynomials cannot be factorised in the form (4). And even when (4) holds, (5) is only a sufficient condition for (2) to have a strong AQLF. But there is no loss of generality in the case $m=1$, because 
then $k_{1}, \ldots, k_{n}$ are the roots of the scalar polynomial $p(\zeta)$ and $(5)$ is both necessary and sufficient for (2) to have a strong AQLF. The following result is proved in section 2:

THEOREM 1. Assume that the characteristic polynomial of the system of difference equations (2) can be factorised in the form (4) and that the constant matrices $k_{1}, k_{2}, \ldots, k_{n}$ in that factorisation satisfy (5). If $\delta_{1}, \delta_{2}, \ldots, \delta_{n}$ is any set of numbers which satisfy the condition

$$
\prod_{v=1}^{n}\left(1-\left\|k_{v}\right\|\right)>\delta_{1}+\delta_{2}+\ldots+\delta_{n}
$$

then (1) has a strong AQLF for all $a_{1}, a_{2}, \ldots, a_{n}$ satisfying (3).

The proof gives a single hermitian form $V$ which serves as a strong AQLF for every equation satisfying (3). The coefficients of $V$ and its constant $\kappa$ depend only on $k_{1}, \ldots, k_{n}$, $\delta_{1}, \ldots, \delta_{n}$. In the general scalar case it seems to be necessary to compute the roots $k_{1}, \ldots, k_{n}$ of $p(\zeta)$ in order to evaluate the left-hand side of (6). But in special cases it may be possible to prove by Sturm's method that these roots are all real numbers between 0 and 1 . Then the lefthand side of (6) is equal to $p(1)$, which can be evaluated without computing $k_{1}, \ldots, k_{n}$. To give some idea of the delicacy of (6) in the scalar case, the following result is proved in section 2:

THEOREM 2. Suppose that (4) is a scalar polynomial and that $\mu$ is the minimum value of $|p(\zeta)|$ on the circle $|\zeta|=1$. If $\delta_{1}+\ldots+\delta_{n} \geqq \mu$, then the trivial solution $x=0$ is not asymptotically stable for some scalar equation (1) which satisfies (3).

Theorem 2 shows that, as a condition on $\delta_{1}, \ldots, \delta_{n},(6)$ is certainly best possible in scalar cases when $\mu=\prod_{v=1}^{n}\left(1-\left|k_{v}\right|\right)$. This is true when all the roots $k_{1}, \ldots, k_{n}$ are positive multiples of a single complex number. With the help of the Möbius transformation theory in [2] Theorem 1 can be made to yield conditions for certain $n$th order differential equations to have a quadratic Lyapunov function. This application is discussed in section 3. The spectral norm used in Theorem 1 is difficult to compute in practice for matrices of high order. A slight modification of Theorem 1 is discussed in section 4, which permits the use of Hölder norms which are much easier to compute.

2. Proof of theorems. Let $R=\left[r_{i j}\right]$ be a real $n \times n$ matrix and let $Q=\left[q_{i j}\right]$ be a block matrix whose elements $q_{i j}$ are all $m \times m$ matrices.

Lemma 1. If $r_{i j} \geqq\left\|q_{i j}\right\|$ for all $i, j$, then $\|R\| \geqq\|Q\|$.

Proof. The matrix spectral norm $\|Q\|$ is related to the euclidean vector norm by

$$
\|Q\|=\sup \{\|Q X\| /\|X\|\},
$$

taken overall vectors $X \neq 0$. By expressing the vectors $X, Q X$ in block formas $X=\operatorname{col}\left(x_{1}, \ldots, x_{n}\right)$, $Q X=\operatorname{col}\left(z_{1}, \ldots, z_{n}\right)$, we can write $z_{i}=\sum_{j=1}^{n} q_{i j} x_{j}$. Then $\left\|z_{i}\right\| \leqq \sum_{j=1}^{n} r_{i j}\left\|x_{j}\right\|$. This gives $\|\eta\| \leqq\|R \xi\|$, where $\xi=\operatorname{col}\left(\left\|x_{1}\right\|, \ldots,\left\|x_{n}\right\|\right), \eta=\operatorname{col}\left(\left\|z_{1}\right\|, \ldots,\left\|z_{n}\right\|\right)$. Since $\|\xi\|=\|X\|$ and $\|\eta\|=\|Q X\|$, it follows that $\|Q X\| \leqq\|R \xi\| \leqq\|R\| .\|X\|$. This and (7) give Lemma 1 . 
Now consider a system of difference equations of the form

$$
Y(t+1)=Q Y(t)
$$

in which $Y(t)$ is a vector and $Q$ is a matrix whose elements are functions of $t, Y(t)$. A positive definite form $V(Y)=Y^{*} P Y$, with constant hermitian matrix $P$, is called a strong AQLF of (8) if there exists a constant $\kappa>1$ such that $\kappa^{2} V(Y(t+1))<V(Y(t))$ for every non-zero solution $Y(t)$ of (8); that is, if $\kappa^{2} V(Q Y)<V(Y)$ for all $t, Y \neq 0$.

LEMMA 2. Suppose that $Q=\left[q_{i j}\right]$ is a block matrix having $\left\|q_{i j}\right\| \leqq r_{i j}$ for all $i, j, t, Y$, where $R=\left[r_{i j}\right]$ is a constant matrix with spectral radius $\rho(R)<1$. Then $(8)$ has a strong AQLF whose matrix $P$ is a diagonal matrix which depends only on $R$.

Proof. We can assume that all the elements of $R$ are positive. (If not, increase them all slightly keeping $\rho(R)<1$.) Then a theorem of Stoer and Witzgall [3] states that $\rho(R)=$ min $\left\|D^{-1} R D\right\|$, taken over all real positive definite diagonal matrices $D$. Let $D_{0}=$ $\operatorname{diag}\left(d_{1}, \ldots, d_{n}\right)$ be the diagonal matrix for which this minimum is attained. Since $\left\|d_{i}^{-1} q_{i j} d_{j}\right\| \leqq$ $d_{i}^{-1} r_{i j} d_{j}$, Lemma 1 gives $\left\|D_{1}^{-1} Q D_{1}\right\| \leqq\left\|D_{0}^{-1} R D_{0}\right\|=\rho(R)$, where $D_{1}$ is the block matrix $\operatorname{diag}\left(d_{1} e, \ldots, d_{n} e\right)$ and $e$ is unit $m \times m$ matrix. If $V(Y)=Y^{*} D_{1}^{-2} Y=\left\|D_{1}^{-1} Y\right\|^{2}$, then

$$
V(Q Y)=\left\|D_{1}^{-1} Q Y\right\|^{2} \leqq\left\|D_{1}^{-1} Q D_{1}\right\|^{2}\left\|D_{1}^{-1} Y\right\|^{2} \leqq \rho(R)^{2} V(Y)<\kappa^{-2} V(Y),
$$

for all $Y \neq 0$ and $1<\kappa<\rho(R)^{-1}$. This shows that $V(Y)$ is a strong AQLF of (8).

Proof of Theorem 1. We first express (1) as a system of the form (8), using a method analogous to that of Skachkov [1]. From any vector function $x(t)$, we can obtain vector functions $y_{1}(t), \ldots, y_{n+1}(t)$ defined iteratively by $y_{1}(t)=x(t)$ and

$$
y_{v+1}(t)=y_{v}(t+1)-k_{v} y_{v}(t) \quad(v=1, \ldots, n),
$$

where $k_{1}, \ldots, k_{n}$ are the matrices in (4). If $S$ is the shift operator defined by the property $S x(t)=x(t+1)$, then (9) gives

$$
y_{v+1}(t)=\left(S e-k_{v}\right)\left(S e-k_{v-1}\right) \ldots\left(S e-k_{1}\right) x(t),
$$

for $v=1, \ldots, n$. In the case $v=n,(10)$ and (4) give

$$
y_{n+1}(t)=p(S) x(t)=x(t+n)+\sum_{v=1}^{n} c_{v} x(t+n-v) .
$$

Replace the left-hand side of this by (9) to get

$$
y_{n}(t+1)-k_{n} y_{n}(t)=x(t+n)+\sum_{v=1}^{n} c_{v} x(t+n-v) .
$$

For $r, s=1,2, \ldots, n$ define $G_{s}^{r}=\sum k_{i_{1}} k_{i_{2}} \ldots k_{i_{r}}$, summed over all sets of $r$ integers $i_{1}, \ldots, i_{r}$ which satisfy $1 \leqq i_{1} \leqq i_{2} \leqq \ldots \leqq i_{r} \leqq s$. In this expression, the sum of all those terms which have $i_{r}<s$ is $G_{s-1}^{r}$ and the sum of all those terms which have $i_{r}=s$ is $G_{s}^{r-1} k_{s}$. Hence

$$
G_{s}^{r}=G_{s-1}^{r}+G_{s}^{r-1} k_{s} \quad(r, s=1, \ldots, n) .
$$


To make this true when $r=1$ or $s=1$, we must define $G_{0}^{v}=0, G_{v}^{0}=e$ for all $v \geqq 1$. We now prove by induction that

$$
x(t+r)=\sum_{s=1}^{r+1} G_{s}^{r+1-s} y_{s}(t),
$$

for $r=0,1, \ldots, n$. If this is true for some $r<n$, then (9) gives

$$
\begin{aligned}
x(t+1+r) & =\sum_{s=1}^{r+1} G_{s}^{r+1-s} y_{s}(t+1) \\
& =\sum_{s=1}^{r+1} G_{s}^{r+1-s}\left\{y_{s+1}(t)+k_{s} y_{s}(t)\right\}
\end{aligned}
$$

Since $G_{0}^{v}=0, G_{v}^{0}=e$, the right-hand side can be rewritten as

$$
\begin{aligned}
x(t+1+r) & =y_{r+2}(t)+\sum_{s=1}^{r+1}\left\{G_{s-1}^{r+2-s}+G_{s}^{r+1-s} k_{s}\right\} y_{s}(t) \\
& =\sum_{s=1}^{r+2} G_{s}^{r+2-s} y_{s}(t),
\end{aligned}
$$

by (12). Hence (13) remains true when $r$ is replaced by $r+1$. Since (13) is trivial for $r=0$, it is true for $r=1, \ldots, n$ by induction. From (11) we get

$$
x(t+n)+\sum_{v=1}^{n} a_{v} x(t+n-v)=y_{n}(t+1)-k_{n} y_{n}(t)+\sum_{v=1}^{n}\left(a_{v}-c_{v}\right) x(t+n-v) .
$$

On the right-hand side, replace $x(t+n-v)$ by (13) to get

$$
x(t+n)+\sum_{v=1}^{n} a_{v} x(t+n-v)=y_{n}(t+1)-k_{n} y_{n}(t)-\sum_{s=1}^{n} F_{s} y_{s}(t),
$$

where $F_{s}=\sum_{\nu=1}^{n+1-s}\left(c_{v}-a_{v}\right) G_{s}^{n+1-s-v}$. This identity shows that $x(t)$ is a solution of $(1)$ if and only if

$$
y_{n}(t+1)=k_{n} y_{n}(t)+\sum_{s=1}^{n} F_{s} y_{s}(t)
$$

This equation and (9) show that (1) is equivalent to a system of the form (8) in which $Y=$ $\operatorname{col}\left(y_{1}, \ldots, y_{n}\right)$ and

$$
Q=\left[\begin{array}{cccccc}
k_{1} & e & 0 & \vdots & 0 & 0 \\
0 & k_{2} & e & \vdots & 0 & 0 \\
\ldots \ldots & \ldots \ldots \ldots \ldots \ldots \ldots \ldots \ldots \ldots \ldots \ldots \ldots \ldots \ldots \ldots & \ldots \ldots \ldots \\
0 & 0 & 0 & \vdots & k_{n-1} & e \\
F_{1} & F_{2} & F_{3} & \vdots & F_{n-1} & k_{n}+F_{n}
\end{array}\right] .
$$


It is understood here that $Q$ is expressed as a function of $t, Y$ by replacing each variable $x(t+r)$ in $F_{v}$ by (13). Now replace $p(\zeta)$ throughout the above argument by the scalar polynomial

$$
\hat{p}(\zeta)=\prod_{v=1}^{n}\left(\zeta-\left\|k_{v}\right\|\right)=\zeta^{n}+\sum_{v=1}^{n} \hat{c}_{v} \zeta^{n-v}
$$

and replace (1) by the scalar difference equation

$$
x(t+n)+\sum_{v=1}^{n}\left(\hat{c}_{v}-\delta_{v}\right) x(t+n-v)=0 .
$$

Then the matrix $G_{s}^{r}$ is replaced by the scalar $\Gamma_{s}^{r}$ which is defined as $\sum\left\|k_{i_{1}}\right\| \ldots\left\|k_{i_{r}}\right\|$ summed over $1 \leqq i_{1} \leqq \ldots \leqq i_{r} \leqq s$. Clearly $\left\|G_{s}^{r}\right\| \leqq \Gamma_{s^{\circ}}^{r}$. The above argument shows that (15) is equivalent to an $n \times n$ system $Y(t+1)=R Y(t)$ having

$$
R=\left[\begin{array}{cccccc}
\left\|k_{1}\right\| & 1 & 0 & \vdots & 0 & 0 \\
0 & \left\|k_{2}\right\| & 1 & \vdots & 0 & 0 \\
\ldots \ldots & \ldots \ldots & \ldots \ldots \ldots \ldots \ldots \ldots \ldots \ldots \ldots \ldots \ldots \ldots \ldots \ldots \ldots \ldots \ldots & 1 \\
0 & 0 & 0 & \vdots & \left\|k_{n-1}\right\| & \left\|k_{n}\right\|+\Phi_{n}
\end{array}\right],
$$

where $\Phi_{s}=\sum_{v=1}^{n+1-s} \delta_{v} \Gamma_{s}^{n+1-v-s}$. This $R$ is constant, and (3) gives

$$
\Phi_{s} \geqq \sum_{v}\left\|c_{v}-a_{v}\right\| \Gamma_{s}^{n+1-v-s} \geqq \sum_{v}\left\|\left(c_{v}-a_{v}\right) G_{s}^{n+1-v-s}\right\| \geqq\left\|F_{s}\right\|,
$$

for all $t, Y, s$; that is, $r_{i j} \geqq\left\|q_{i j}\right\|$ for all $t, Y, i, j$, where $\left[r_{i j}\right]=R,\left[q_{i j}\right]=Q$. This establishes one condition of Lemma 2, and the other condition $\rho(R)<1$ will now be verified.

Since the system $Y(t+1)=R Y(t)$ is equivalent to (15), the eigenvalues of $R$ are the characteristic roots of (15). These are the roots of the equation $\hat{p}(\zeta)-d(\zeta)=0$, where $d(\zeta)=\sum_{v=1}^{n} \delta_{v} \zeta^{n-v}$. For all complex $\zeta$ with $|\zeta|=1,(14)$ and (6) give

$$
|\hat{p}(\zeta)| \geqq \prod_{v=1}^{n}\left(1-\left\|k_{v}\right\|\right)>\sum_{v=1}^{n} \delta_{v} \geqq|d(\zeta)| .
$$

Since the roots $\left\|k_{1}\right\|, \ldots,\left\|k_{n}\right\|$ of $\hat{p}(\zeta)=0$ all lie inside the circle $|\zeta|=1$, the same is true of the roots of $\hat{p}(\zeta)-d(\zeta)=0$, by Rouché's theorem. Hence $\rho(R)<1$.

Lemma 2 now gives a diagonal form $V(Y)$ which is a strong AQLF of (8). When we replace each variable $y_{v}$ in $V(Y)$ by the corresponding expression (10) we obtain a hermitian form in the variables $x, S x, S^{2} x, \ldots, S^{n-1} x$ which is a strong AQLF of (1). This completes the proof of Theorem 1.

Proof of Theorem 2. By definition, $\mu=|p(w)|$ for some complex $w$ with $|w|=1$. If $v$ is defined by $p(w)=v\left(\delta_{1}+\ldots+\delta_{n}\right)$, then $|v|=\mu /\left(\delta_{1}+\ldots+\delta_{n}\right) \leqq 1$. Now put $a_{v}=c_{v}-w^{v-n} \delta_{v} v$ in (1). Then (3) holds and (1) has the solution $x(t)=\lambda w^{t}$, where $\lambda$ is an arbitrary constant. Since $|x(t)|=|\lambda|$ for all $t$, the trivial solution of (1) cannot be asymptotically stable. This proves Theorem 2 . 
3. Application to differential equations. Any Möbius transformation $\zeta=u(z)=$ $(\alpha z+\beta) /(\gamma z+\delta)$ can be used to associate with (1) a system of differential equations as follows. From the characteristic polynomial $f(z)=z^{n}+\sum_{v=1}^{n} a_{v} z^{n-v}$ of (1) we get a new polynomial $g(\zeta)$ defined by

$$
g(\zeta)=(\alpha \delta-\beta \gamma)^{-n / 2}(\alpha-\gamma \zeta)^{n} f\left(u^{-1}(\zeta)\right),
$$

where $z=u^{-1}(\zeta)=(\delta \zeta-\beta) /(\alpha-\gamma \zeta)$ is the inverse of $\zeta=u(z)$. This can be expanded in the form $g(\zeta)=\sum_{\nu=0}^{n} h_{v} \zeta^{n-v}$, where the matrices $h_{0}, \ldots, h_{n}$ are linear combinations of $e, a_{1}, \ldots, a_{n}$ with scalar coefficients. Since $a_{1}, \ldots, a_{n}$ in (1) are functions of $t, x(t), \ldots, x(t+n-1)$, the same is true of $h_{0}, \ldots, h_{n}$. These are now made into functions of $t, x, D x, \ldots, D^{n-1} x$ by replacing $x(t+v)$ by $D^{v} x$ for $v=1, \ldots, n-1$. If the matrix $h_{0}$ is invertible, then $h_{0}^{-1} g(\zeta)$ is the characteristic polynomial of the system of differential equations

$$
D^{n} x+b_{1} D^{n-1} x+b_{2} D^{n-2} x+\ldots+b_{n} x=0,
$$

in which $D$ denotes $d / d t$ and $b_{v}=h_{0}^{-1} h_{v}$. Conversely, if we are given any system of the form (17), we can recover the corresponding system (1) as follows. Replace $\zeta$ by $u(z)$ in (16) to get

$$
\begin{aligned}
(\alpha \delta-\beta \gamma)^{n / 2} f(z) & =(\gamma z+\delta)^{n} g(u(z)) \\
& =h_{0}(\alpha z+\beta)^{n}+\sum_{v=1}^{n} h_{0} b_{v}(\alpha z+\beta)^{n-v}(\gamma z+\delta)^{v}
\end{aligned}
$$

Equate the coefficients of like powers of $z$ on both sides to obtain

$$
\begin{aligned}
& h_{0}=(\alpha \delta-\beta \gamma)^{n / 2}\left(\alpha^{n} e+\sum_{v=1}^{n} \alpha^{n-v} \gamma^{v} b_{v}\right)^{-1}, \\
& a_{s}=\left(\alpha^{n} e+\sum_{v=1}^{n} \alpha^{n-v} \gamma^{v} b_{v}\right)^{-1}\left(\Lambda_{s}^{0} e+\sum_{v=1}^{n} \Lambda_{s}^{v} b_{v}\right),
\end{aligned}
$$

where $\Lambda_{s}^{v}$ is the coefficient of $z^{n-s}$ in the expansion of $(\alpha z+\beta)^{n-v}(\gamma z+\delta)^{v}$. That is, any system of differential equations of the form (17) is associated with a system of difference equations of the form (1), whose coefficients $a_{1}, \ldots, a_{n}$ are given by (18), provided that the inverse matrix in (18) exists.

By a strong AQLF of $(17)$ is meant a positive definite hermitian form $V\left(x, D x, \ldots, D^{n-1} x\right)$ with constant coefficients such that $d V / d t<-\kappa V$ for some constant $\kappa>0$ and every non-zero solution $x(t)$ of (17). The existence of a strong AQLF is a sufficient condition for the global asymptotic stability of the trivial solution $x=0$ of (17). It has been proved elsewhere that if the coefficients $\alpha, \beta, \gamma, \delta$ of $u(z)$ are independent of $t$ and, if $\zeta=u(z)$ maps the disc $|z|<1$ into the half plane $\operatorname{Re} \zeta<0$, then the existence of a strong AQLF of (1) implies the existence of a strong AQLF of (17) (see [2], Theorem 7). This enables us to deduce conditions for (17) to have a strong AQLF from the conditions given in Theorem 1 for (1) to have a strong AQLF. 
The requirement that $\zeta=u(z)$ map $|z|<1$ into $\operatorname{Re} \zeta<0$ is certainly satisfied if $\alpha, \beta, \gamma, \delta$ are real and $0<-u(0)<\infty, 0 \leqq-u(1) \leqq \infty, 0 \leqq-u(-1) \leqq \infty$; that is, if

$$
0<\frac{-\beta}{\delta}<\infty, \quad 0 \leqq \frac{-(\alpha+\beta)}{\gamma+\delta} \leqq \infty, \quad 0 \leqq \frac{\alpha-\beta}{\delta-\gamma} \leqq \infty .
$$

A simple example satisfying these conditions is $u(z)=(z+1) /(z-1)$. We have now shown that, if $\alpha, \beta, \gamma, \delta$ are any real constants satisfying $\alpha \delta \neq \beta \gamma$ and (19) and if the matrices $a_{1}, \ldots, a_{n}$ defined by (18) satisfy the conditions of Theorem 1, then the system (17) has a strong AQLF. Because of the simplicity of Theorem 1, this stability condition for differential equations could be useful in practice. The computation of the spectral norms in (3) and of the inverse matrix in (18) present no difficulties in the scalar case $m=1$.

4. On Hölder norms. The Hölder norms of any vector $X=\operatorname{col}\left(x_{1}, \ldots, x_{\mathrm{r}}\right)$ are $\|X\|_{\sigma}=$ $\left(\sum_{v=1}^{r}\left|x_{v}\right|^{\sigma}\right)^{1 / \sigma}$ for $1 \leqq \sigma<\infty$ and $\|X\|_{\infty}=\max \left(\left|x_{1}\right|, \ldots,\left|x_{r}\right|\right)$. The corresponding matrix norms $\|Q\|_{\sigma},\|Q\|_{\infty}$ are defined by (7). The spectral norm used in Theorem 1 is the special case $\sigma=2$. It is well known that

$$
\|Q\|_{1}=\max _{1 \leqq j \leqq r}\left(\sum_{i=1}^{r}\left|\theta_{i j}\right|\right), \quad\|Q\|_{\infty}=\max _{1 \leqq i \leqq r}\left(\sum_{j=1}^{r}\left|\theta_{i j}\right|\right),
$$

where $\left[\theta_{i j}\right]=Q$ is an $r \times r$ matrix. These two norms are particularly useful for practical computation. The theorem of Stoer and Witzgall used in the proof of Lemma 2 is valid for any Hölder norm with $1 \leqq \sigma \leqq \infty$. If we take the norm throughout section 2 to be a Hölder norm, then all remains valid except that Lemma 2 yields a Lyapunov function $V(Y)=\left\|D_{1}^{-1} Y\right\|^{2}$ which is not a quadratic form when $\sigma \neq 2$. It follows that, if we take the norm in the statement of Theorem 1 to be a Hölder norm with $\sigma \neq 2$, then, instead of a quadratic Lyapunov function, the proof yields a Lyapunov function of the form $\|M X\|^{2}$, where $M$ is a constant invertible matrix and $X=\operatorname{col}(x(t), x(t+1), \ldots, x(t+n-1))$. From the existence of this Lyapunov function we can deduce as before that the trivial solution of (1) is globally asymptotically stable. But the application to differential equations discussed in section 3 appears to be valid only when the spectral norm is used, because the Möbius transformation theory in [2] was only proved for quadratic Lyapunov functions.

\section{REFERENCES}

1. B. N. Skachkov, On the stability of the zero solution of a class of $n$th order differential equations (Russian), Differencial'nye Uravnenija 1 (1965), 171-175. English translation: Differential Equations 1 (1965), 126-129. 143-151.

2. R. A. Smith, Möbius transformations in stability theory, Proc. Cambridge Phil. Soc. 68 (1970),

3. J. Stoer and C. Witzgall, Transformation by diagonal matrices in a normed space, Numerische Math. 4 (1962), 158-171.

UNIVERSITY OF DURHAM

DuRHAM, ENGLAND 